Artículo

\title{
Análisis de los indicadores de competitividad de las exportaciones de fresa mexicana
}

\author{
Laura Cecilia Ramírez Padrón ${ }^{1}$ \\ Ignacio Caamal Cauich ${ }^{1 \S}$ \\ Verna Gricel Pat Fernández ${ }^{1}$ \\ David Martínez Luis ${ }^{2}$ \\ Alberto Pérez Fernández ${ }^{2}$
}

${ }^{1}$ Universidad Autónoma Chapingo. Carretera México-Texcoco km 38.5. Texcoco, Estado de México. CP. 56230. (laura.ramirez.lr826@gmail.com; gricelpat@hotmail.com). ${ }^{2}$ Universidad Autónoma del Carmen. Calle 56, No. 4, Esq. Avenida Concordia, Col. Benito Juárez, Ciudad del Carmen, Campeche, México. CP. 24180. (Luis_david25@ hotmail.com; albertopefe@gmail.com).

${ }^{\S}$ Autor para correspondencia: icaamal82@yahoo.com.mx.

\section{Resumen}

Los principales países productores de fresa son China, Estados Unidos de América, México, Turquía y Egipto, los cuales en conjunto aportan más del setenta por ciento del volumen total de la producción de fresa en el mundo y por otro lado, los principales países exportadores son España, Estados Unidos de América, México, Países Bajos y Bélgica, destacando México como tercer productor y exportador de fresa en el mundo. El objetivo del trabajo es analizar la competitividad de las exportaciones de la fresa mexicana en el mercado mundial exportador, con el cálculo de los índices de exportación, de ventaja relativa de exportaciones y de ventaja comparativa revelada, con información estadística de las variables de producción, exportación e importación, tomada de las bases de datos de FAS-USDA y FAOSTAT. Los índices obtenidos de exportación, mayor que cero, ventaja relativa de las exportaciones, mayor que uno y ventaja comparativa revelada, mayor que uno, reflejan que la producción de fresa mexicana tiene una especialización exportadora y las exportaciones de fresa mexicana son competitivas en el mercado exportador. Asimismo, los índices calculados positivos para los principales países exportadores fueron mayores para España, seguido de México y por último de Estados Unidos de América, reflejando especialización exportadora y competitividad de las exportaciones diferenciadas.

Palabras clave: competitividad, exportación, importación, producción.

Recibido: marzo de 2020

Aceptado: mayo de 2020 


\section{Introducción}

La fresa es una fruta muy apreciada por su agradable sabor agridulce, su atractivo aroma, sus propiedades nutricionales y cualidades medicinales. La fresa contiene vitamina $\mathrm{C}$, taninos, flavonoides, antocianinas, catequina, quercetina y kaempferol, ácidos orgánicos (cítrico, málico, oxálico, salicílico y elágico), minerales (potasio, fósforo, calcio, sodio y hierro), pigmentos y aceite esencial, estos compuestos tienen un potente poder antioxidante y ayudan a disminuir el riesgo de enfermedades cardiovasculares, mejoran la función endotelial vascular y disminuyen la trombosis (Özcan, 2007; Pinto et al., 2008; Beattie et al., 2005, citados por Restrepo et al., 2009).

La composición química de la fresa es de $89.6 \%$ de agua, incluye hidratos de carbono, proteínas, lípidos y fibra; y, además, la fresa contiene 35 calorías por cada cien gramos (Chordi, 2013). La producción mundial de fresa en el 2016 fue de $9059817 \mathrm{t}$, siendo los principales países productores China con 3779831 t, Estados Unidos de América (EE. UU.) con 1431050 t, México con 468 248 t, Turquía con 415150 t y Egipto con 378960 t, los cuales aportaron $71.45 \%$ del total de la fresa producida en el mundo (FAOSTAT, 2019).

Por otro lado, las exportaciones mundiales de fresa en 2016 ascendieron a $853213 \mathrm{t}$, siendo los principales exportadores España con 314256 t, Estados Unidos de América con 134406 t, México con 102631 t, Países Bajos con 48425 t y Bélgica con 40718 t, exportadas respectivamente, los cuales aportaron $75.06 \%$ de las exportaciones mundiales de fresa. México fue el tercer productor y exportador de fresa en el mercado mundial (FAOSTAT, 2019).

El coeficiente de exportación mundial de la fresa es de 9.4\%, señalando que es un producto de mayor consumo doméstico. La producción mundial de fresa fue de 3392922 t en 1994 y de 9059 817 t en 2016, con una tasa de crecimiento de $167.02 \%$. La producción de fresa en China fue de $750000 \mathrm{t}$ en 1994 y de $3779831 \mathrm{t}$ en 2016, con una tasa de crecimiento de $403.98 \%$, en EE. UU fue de $748330 \mathrm{t}$ en 1994 y de $1431050 \mathrm{t}$ en 2016, con una tasa de crecimiento de $91.23 \%$, en México fue de 95006 t en 1994 y de 468248 t en 2016, con una tasa de crecimiento de 392.86\%, en Turquía fue de 65000 t en 1994 y de 415150 t en 2016, con una tasa de crecimiento de 538.69\% y en Egipto la producción fue de 27000 t en 1994 y de 378960 t en 2016, con una tasa de crecimiento de $1303.56 \%$ (FAOSTAT, 2019).

Las exportaciones en el mundo de fresa fueron de $392660 \mathrm{t}$ en 1994 y de $862830 \mathrm{t}$ en 2016, con una tasa de crecimiento de 119.74\%. Las exportaciones en España fueron de $159508 \mathrm{t}$ en $1994 \mathrm{y}$ de $314256 \mathrm{t}$ en 2016, con una tasa de crecimiento de 97.02\%, en EE. UU. fueron de $57450 \mathrm{t}$ en 1994 y de 134406 t en 2016, con una tasa de crecimiento de 133.95\%, en México fueron de 19 471 t en 1994 y de 102631 t en 2016, con una tasa de crecimiento de 427.10\%, en Países Bajos fueron de $15785 \mathrm{t}$ en 1994 y de $48425 \mathrm{t}$ en 2016, con una tasa de crecimiento de $206.78 \%$ y en Bélgica, las exportaciones a partir del 2000 fueron de 39553 t y de 40718 t en 2016, con una tasa de crecimiento de 2.95\%. El destino de las exportaciones de fresa de México es Estados Unidos de América, el cual recibe $99.8 \%$ de las exportaciones totales de México (FAOSTAT, 2019).

Las tasas de crecimiento positivas y altas de la producción y exportación mundial y en los principales países productores y exportadores, refleja que el cultivo de la fresa se encuentra en expansión y es competitivo. 
El objetivo del trabajo es analizar la competitividad de las exportaciones de fresa de México, con base en los índices de exportación, ventaja relativa de las exportaciones y ventaja comparativa revelada y además, comparar la competitividad de las exportaciones de fresa mexicana con la competitividad de las exportaciones de los otros principales países exportadores de fresa en el mundo, España y Estados Unidos de América. La hipótesis de trabajo plantea que las exportaciones de fresa mexicana son competitivas en el mercado exportador y los indicadores de competitividad y ventaja comparativa revelada son positivos y crecientes.

\section{Marco teórico}

La participación total de mercado de un país en un sector dado de actividad comercial internacional es conocida como competitividad global, involucrando el crecimiento de todos los sectores agregados y la penetración de los países en los mercados internacionales (Mortimore et al., 1997). La competitividad de un país se presenta cuando dicho país, bajo condiciones de mercado libres y justas, produzca bienes y servicios que superen el promedio producido en los mercados internacionales (OCDE, 1992).

La competitividad desde sus orígenes ha estado ligada al comercio internacional, entendida como la capacidad de una determinada nación de insertarse exitosamente en el mercado internacional (Daza, 2014), por lo que la medición de las ventajas competitivas mediante el análisis de los flujos comerciales, importaciones y exportaciones de un país en el mercado internacional, es pertinente. La medición de la participación de un país en los mercados se puede realizar a través de indicadores indirectos tales como el índice de ventaja comparativa revelada.

El cual se desprende de la ventaja comparativa, que es una ventaja natural, y se presenta cuando se produce mejor y con menor costo relativo en comparación con otro país, en donde los países tienden a especializarse en la producción y exportación de aquellos productos con menor costo relativo (Vargas, 2014). La teoría de la ventaja comparativa se refiere a la mayor eficiencia en la producción de algunos bienes nacionales, con menor costo comparativo de producción, llevando a un intercambio más ventajoso, siendo la base de la exportación de los países que participan en el comercio internacional (Salazar, 2015).

La ventaja comparativa entre países se relaciona con la eficiencia en la producción y el país con menor costo de oportunidad será aquel que sea más eficiente y con ello tenga ventaja comparativa sobre los otros (Arias y Segura, 2004). La ventaja comparativa sirve para el estudio de los términos de intercambio entre dos o más países y entre un país determinado y el resto del mundo (Ayvar $e t$ al., 2018), el país en el cual el valor agregado es mayor al valor de los recursos domésticos presentará ventajas comparativas (Contreras, 1999).

La ventaja comparativa se puede evaluar con los indicadores de ventaja comparativa revelada. Balassa (1965), fue el primero en acuñar el término de ventaja comparativa revelada para indicar que las ventajas comparativas pueden ser reveladas por los flujos de comercio de mercancías, el intercambio refleja costos relativos y otros factores que inciden en las transacciones comerciales (Heredia y Huarachi, 2009) y además mide el grado de importancia de un producto.

Dentro de las exportaciones de un mercado a otro mercado versus la importancia de las exportaciones del mismo producto en las exportaciones del mismo producto hacia el mundo (De Pablo y Giacinti, 2011). Asimismo, para la medición de la competitividad de las exportaciones en 
el mercado internacional se puede utilizar el indicador de ventaja comparativa revelada (IVCR) establecido por Vollrath en donde se examina la competitividad internacional a través de mediciones, que utilizan datos de comercio como exportaciones e importaciones y de esta forma, incorpora los efectos de oferta y demanda relativa de bienes (González, 2017), mediante el índice de ventaja relativa de exportación (IVRE) y el índice de ventaja comparativa revelada (IVCR). La ventaja relativa de exportación (VRE) indica las ventajas de un país en referencia a sus exportaciones totales, así como en su participación al resto del mundo y se mide a través del índice de ventaja comparativa revelada.

El valor del IVRE sugerirá la existencia de ventaja comparativa o especialización en las exportaciones de cierto producto (Ávila y González, 2012), un valor positivo, mayor que cero, indica la existencia de una ventaja comparativa revelada y mientras más elevado sea el valor del índice mayor será el grado de especialización y revelará una mayor competitividad, la tendencia creciente a lo largo de los años indicará mayor competitividad en el mercado (Avendaño, 2008).

Los índices de competitividad y ventaja comparativa de las exportaciones (coeficiente o índice de exportación, índice de ventaja relativa de las exportaciones e índice de ventaja comparativa revelada) son útiles para realizar análisis a nivel de agregados económicos, y permiten identificar la existencia de ventaja comparativa, con base en recursos internos (naturales, mano de obra y capital), especialización exportadora y competitividad en el mercado exportador (Vargas, 2014; González, 2017).

El índice de ventaja comparativa revelada (IVCR), es de utilidad para evaluar la especialización en la exportación de un producto, en este caso la fresa, en relación con la totalidad de productos exportados por un país en comparación con el mundo (De Pablo y Giacinti, 2011), el IVCR puede adoptar valores positivos o negativos, reflejando la posición de competitividad de un país en el mercado internacional con el producto en comercio.

Investigaciones realizadas por Arias y Segura (2004) refieren que cuando el IVCR toma valores mayores a cero, el producto en comercio cuenta con una ventaja comparativa revelada debido a que las exportaciones del país exceden las importaciones, y cuanto más alto sea el valor del IVCR del bien, tendrá mayor competitividad en el mercado internacional.

\section{Materiales y métodos}

Los índices de competitividad y ventaja comparativa revelada calculados son el coeficiente de exportación, la ventaja comparativa relativa de exportación y la ventaja comparativa revelada para España, Estados Unidos de América y México, debido a que son los principales países exportadores de fresa.

\section{Procedimientos de cálculo}

El coeficiente de exportación (CE) indica la proporción de la producción nacional que se exporta y entre más alto sea el valor del coeficiente mayor será la competitividad del producto en comercio y se calcula con la fórmula siguiente: $\mathrm{CE}=\left(\frac{\mathrm{X}_{\mathrm{TX}}}{\mathrm{Q}_{\mathrm{TX}}}\right) 100$; donde: $\mathrm{CE}=$ coeficiente de exportación; $\mathrm{X}_{\mathrm{TX}}=$ exportaciones totales del producto $\mathrm{x} ; \mathrm{Q}_{\mathrm{TX}}=$ producción total del producto $\mathrm{x}$. 
El índice de ventaja relativa de exportación (IVRE) refleja las ventajas o desventajas en las exportaciones de un país en el mundo (Ávila y González, 2012) y se obtiene de la manera siguiente: $\operatorname{IVRE}_{\mathrm{a}}^{\mathrm{i}}=\left(\mathrm{X}_{\mathrm{a}}^{\mathrm{i}} / \mathrm{X}_{\mathrm{n}}^{\mathrm{i}}\right) /\left(\mathrm{X}_{\mathrm{a}}^{\mathrm{r}} / \mathrm{X}_{\mathrm{n}}^{\mathrm{r}}\right)$, donde: $\operatorname{IVRE}_{\mathrm{a}}^{\mathrm{i}}=$ índice de ventaja relativa de exportación del producto a en el país $\mathrm{i}$; $\mathrm{X}_{\mathrm{a}}^{\mathrm{i}}=$ valor de las exportaciones del producto a en el país $\mathrm{i}$; $\mathrm{X}_{\mathrm{n}}^{\mathrm{i}}=$ valor de las exportaciones totales menos el producto a en el país $\mathrm{i} ; \mathrm{X}_{\mathrm{a}}^{\mathrm{r}}=$ valor de las exportaciones del producto a en el mundo (menos el país i); $\mathrm{X}_{\mathrm{n}}^{\mathrm{r}}=$ valor de las exportaciones totales (menos el producto a) en el mundo (menos el país i).

El índice de ventaja comparativa revelada (IVCR), refleja la especialización y competitividad de la exportación de un producto con relación al resto de productos con el mundo (Vollrath, 1991) y se calcula con la fórmula siguiente: $\operatorname{IVCR}_{\mathrm{a}}^{\mathrm{i}}=\operatorname{IVCE}_{\mathrm{a}}^{\mathrm{i}}-\operatorname{IVCI}_{\mathrm{a}}^{\mathrm{i}}$, donde: $\operatorname{IVCE}_{\mathrm{a}}^{\mathrm{i}}=$ índice de ventaja comparativa revelada de las exportaciones, la cual se obtiene de la siguiente forma: $\operatorname{IVCE}_{\mathrm{a}}^{\mathrm{i}}=$ $\ln \left[\left(\mathrm{X}_{\mathrm{a}}^{\mathrm{i}} / \mathrm{X}_{\mathrm{n}}^{\mathrm{i}}\right) /\left(\mathrm{X}_{\mathrm{a}}^{\mathrm{r}} / \mathrm{X}_{\mathrm{n}}^{\mathrm{r}}\right)\right]$, donde $\mathrm{IVCl}_{\mathrm{a}}^{\mathrm{i}}=$ índice de ventaja comparativa revelada de las importaciones y se calcula mediante la siguiente ecuación: $\operatorname{IVCI}_{a}^{i}=\ln \left[\left(M_{a}^{i} / M_{n}^{i}\right) /\left(M_{a}^{r} / M_{n}^{r}\right)\right]$. Donde: X y $M$ son exportaciones e importaciones respectivamente, $\mathrm{i}=$ es el país para el cual se está realizando el análisis, $\mathrm{r}=$ se refiere al resto del mundo excepto el país de análisis (país i), a= es el bien o mercancía que se está investigando y $\mathrm{n}=$ involucra el comercio de todas las mercancías menos el bien en análisis (bien a).

\section{Variables}

Para el cálculo del coeficiente de exportación se usaron datos de exportación y producción de fresa en toneladas, para obtener el índice de ventaja relativa de exportación y del índice de ventaja comparativa revelada de Vollrath, se utilizaron datos de comercio tales como valor de las exportaciones de fresa en México, valor de las exportaciones totales de productos agrícolas (menos la fresa) en México, valor de las exportaciones de fresa en el mundo (menos México).

Valor de las exportaciones totales de productos agrícolas (menos fresa) en el mundo (menos México), valor de las importaciones de fresa en México, valor de las importaciones totales de productos agrícolas (menos la fresa) en México, valor de las importaciones de fresa en el mundo (menos México), valor de las importaciones totales de productos agrícolas (menos fresa) en el mundo (menos México). Las fuentes de los datos estadísticos utilizados fueron la Foreign Agricultural Service's Global Agricultural Trade System (FAS-USDA) y la Organización de las Naciones Unidas para la Alimentación y la Agricultura (FAOSTAT), el período consultado fue de 1994 a 2016.

\section{Resultados y discusión}

Los principales exportadores de fresa en el mundo son España, Estados Unidos de América y México, los cuales han venido aportando de manera creciente los mayores volúmenes de producción y exportación de fresa a lo largo del período comprendido de 1994 a 2016 (FAOSTAT, 2019). En 1994, España con una producción de 282200 t y un volumen exportado de 159508 toneladas, tuvo un coeficiente de exportación de $56.5 \%$, lo que refleja que más de la mitad de la producción fue destinada a la exportación en 2016. 
La producción de fresa en España fue de 377596 t y el volumen exportado de 314256 t (Figura 1 y 2), con un coeficiente de exportación de más de $80 \%$, lo que indica que España es un país altamente competitivo en la producción y exportación de fresa, puesto que una alta proporción de la producción se destina al mercado externo (FAOSTAT, 2019).

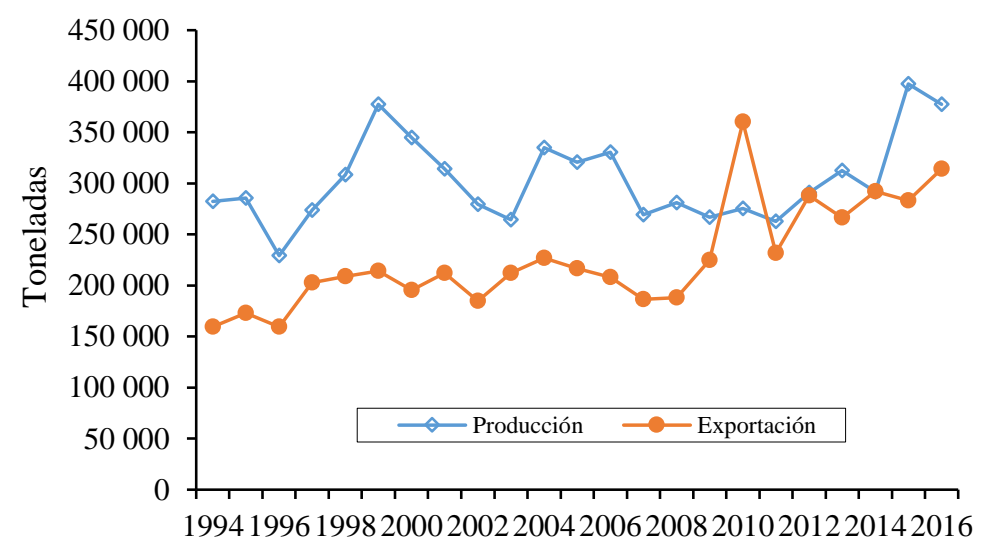

Figura 1. Producción y exportación de fresa en España, 1994-2016. Elaborado con datos de FAOSTAT (2019).

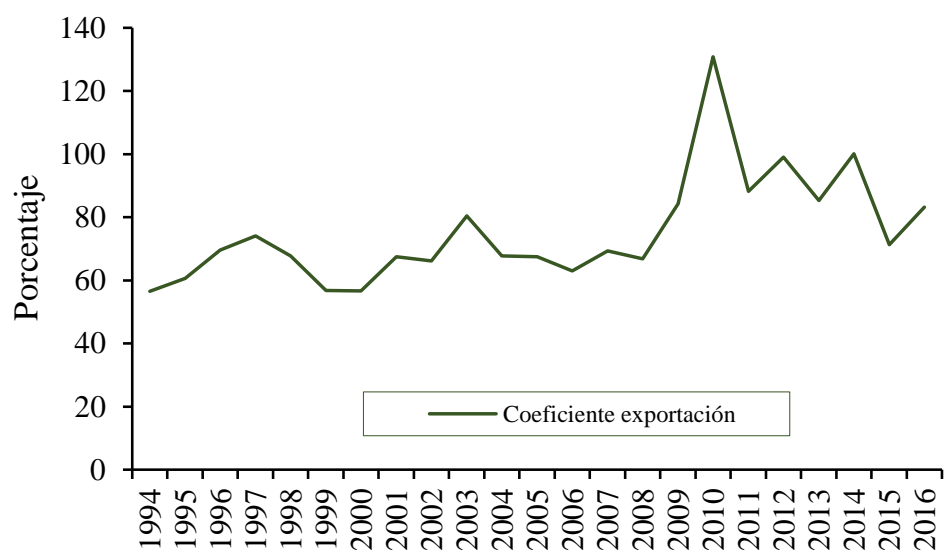

Figura 2. Coeficiente de exportación de fresa en España, 1994-2016. Elaborado con datos de FAOSTAT (2019).

Estados Unidos de América en 1994 tuvo una producción de 748330 t y el volumen exportado fue de 57450 t, con un coeficiente de exportación de 7.7\%, lo que refleja que una baja proporción de la producción se destinó a la exportación; mientras que en 2016 la producción fue de 1431 050 t y las exportaciones ascendieron a 134406 t, con un coeficiente de exportación de 9.3\% (Figura 3 y 4), reflejando un bajo coeficiente de exportación y volumen exportado, el cual se debe a que una gran parte de la producción se destina a satisfacer la demanda del mercado interno (FAOSTAT, 2019). 


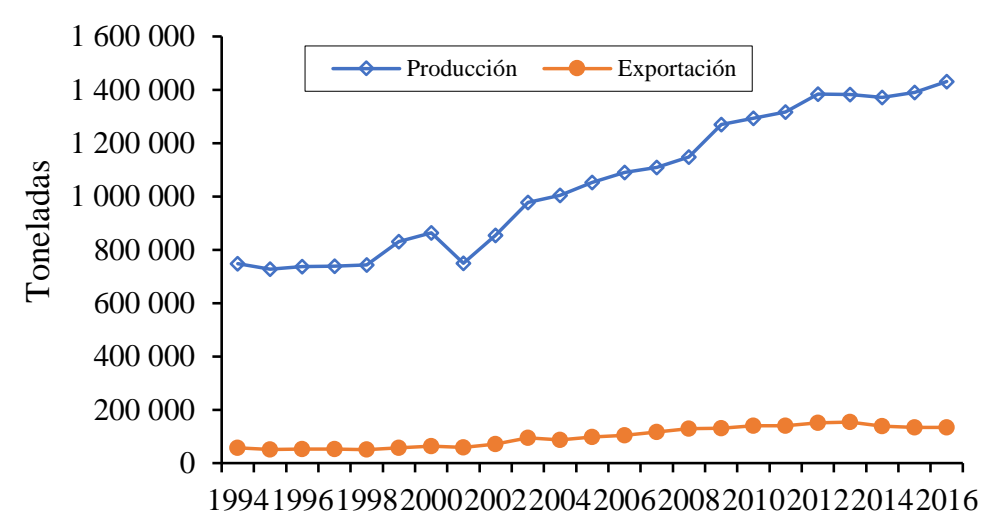

Figura 3. Producción y exportación de fresa en Estados Unidos de América 1994-2016. Elaborado con datos de FAOSTAT (2019).

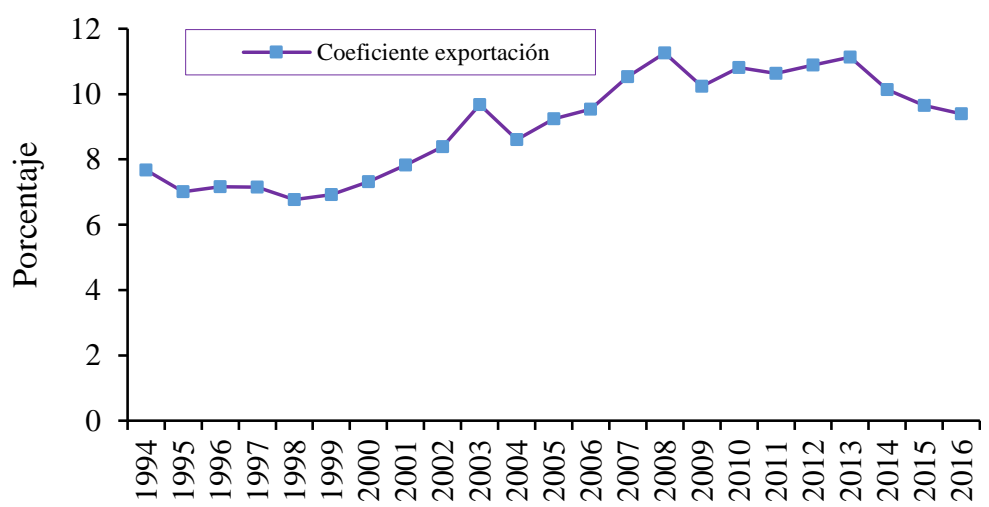

Figura 4. Coeficiente de exportación de fresa en Estados Unidos de América 1994-2016. Elaborado con datos de FAOSTAT (2019).

México, que es otro de los principales productores y exportadores de fresa a nivel mundial, en 1994 produjo 95006 t y exportó 19471 t y el coeficiente de exportación fue de $20.49 \%$, en 2016 la producción de fresa en México se colocó en 468248 t y el volumen exportado fue de 102631 toneladas, con lo que el valor del coeficiente de exportación fue de $21.9 \%$ (Figura 5 y 6), indicando que una buena parte de la producción se destinó al mercado externo (FAOSTAT, 2019).

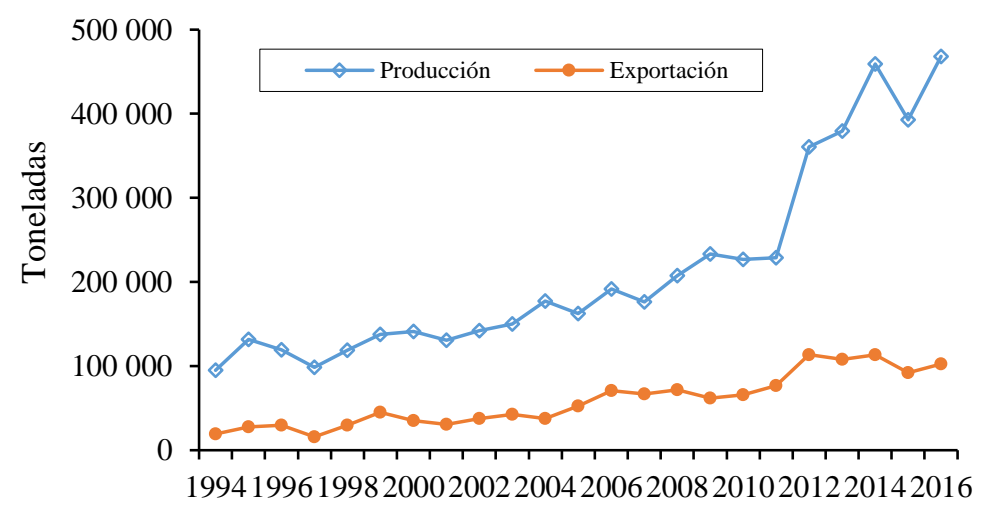

Figura 5. Producción y exportación de fresa en México, 1994-2016. Elaborado con datos de FAOSTAT (2019). 


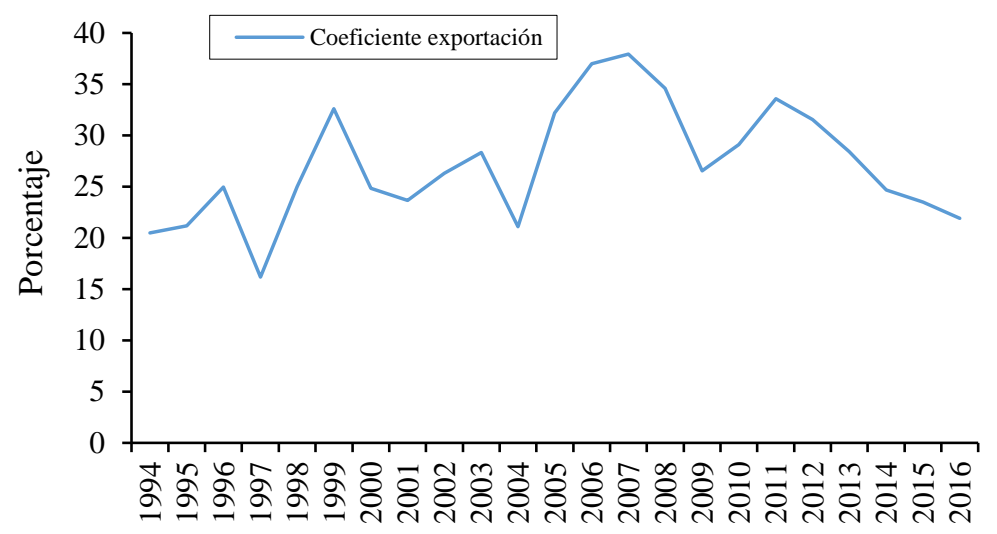

Figura 6. Coeficiente de exportación de fresa de México, 1994-2016. Elaborado con datos de FAOSTAT (2019).

En el periodo de 1994 a 2016 el coeficiente de exportación de España fue en promedio de 75.3\%, Estados Unidos de América de 9\% y México de 27.2\% (FAOSTAT, 2019). Los valores de los índices reflejan especialización exportadora de España, México y Estados Unidos de América, coincidiendo con los resultados obtenidos para México de otros estudios similares (Ayvar y González, 2011).

España, primer exportador de fresa a nivel mundial, durante el periodo de 1994 a 2016 tuvo un índice de ventaja relativa de exportación con valores mayores a uno, que lo ubica como el país con mayor grado de especialización y de mayor competitividad en la exportación de la fresa, en 1994 el valor del IVRE fue de 1.105 y en 2016 de 1.09, con una tendencia ligeramente decreciente, conservando la posición de país con mayor ventaja relativa de exportación de fresa (Figura 7).

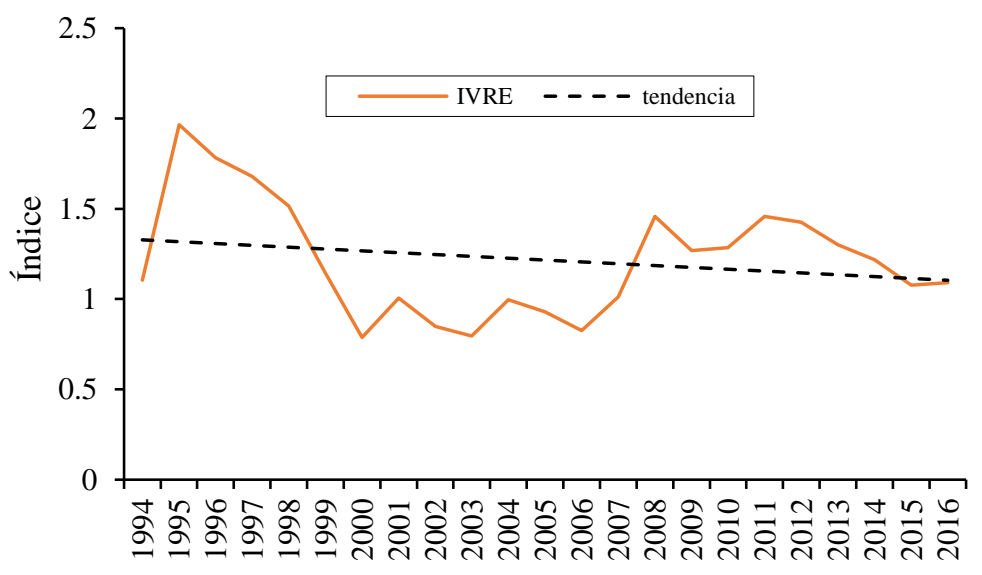

Figura 7. Índice de ventaja relativa de exportación de fresa, 1994-2016. España. Elaborado con datos de FAOSTAT (2019); FAS-USDA (2019).

En cuanto al índice de ventaja comparativa revelada el valor máximo lo alcanzó en 1994 con 6.1 y posteriormente bajó en 2015 a 2.93 y en 2016 a 2.86; no obstante, a pesar de haberse mostrado una tendencia decreciente en el IVCR (Figura 8). 


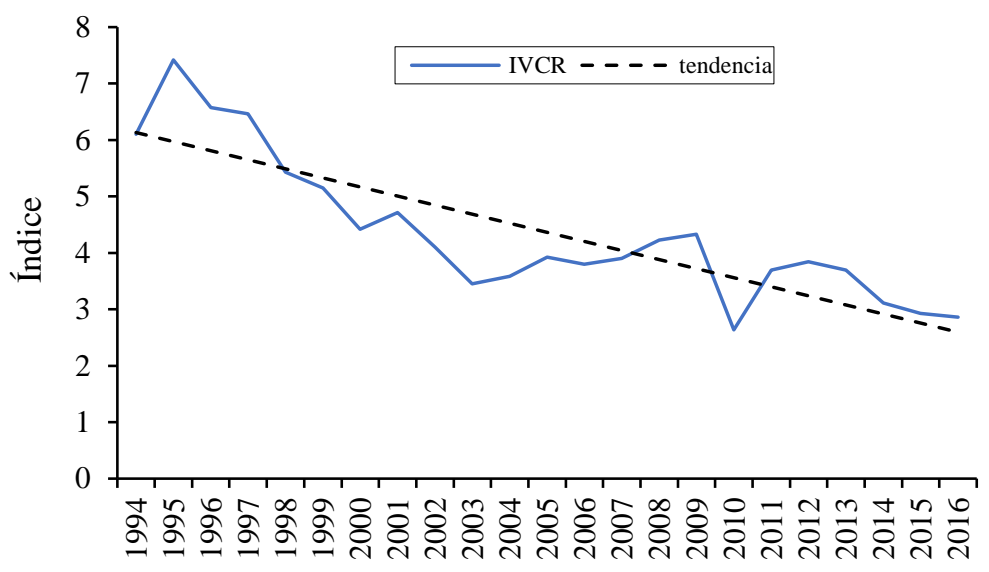

Figura 8. Índice de ventaja comparativa revelada de fresa, 1994-2016. España. Elaborado con datos de FAOSTAT (2019); FAS-USDA (2019).

Los valores que muestra España son superiores a los de Estados Unidos de América y de México. España es el país con mayor competitividad y de mayor especialización en la exportación de fresa, en el mercado de la fresa a nivel internacional. El índice de ventaja relativa de exportación de fresa producida en Estados Unidos de América en 1994 fue de 0 y en 2016 de 0.45433, el cual tuvo mayor relevancia a partir de 2004 hasta 2016.

Donde los valores del IVRE oscilaron entre 0.0108 a 0.3339 , los cuales mantuvieron una tendencia creciente, indicando con ello que la fresa producida en Estados Unidos de América es cada vez más competitiva a nivel internacional, con exportaciones y grado de especialización exportadora crecientes (Figura 9). En el caso del IVCR de la fresa estadounidense muestra una mayor variación, en 2003 tuvo el valor más alto con 3.51 y en 2016 el valor alcanzado fue de 0.45 (Figura 10), lo que indica que tiene un nivel de competitividad y grado de especialización en la exportación de fresa decreciente en el mercado internacional.

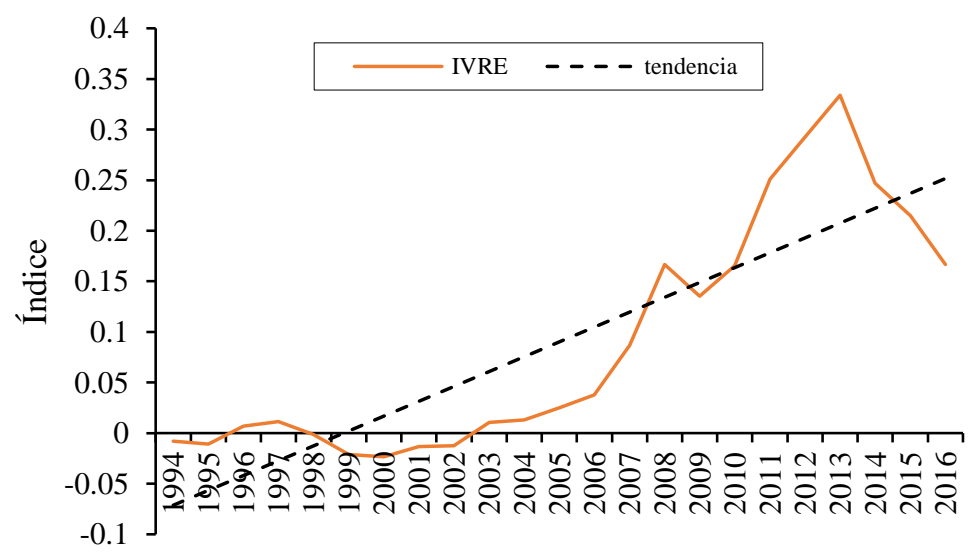

Figura 9. Índice de ventaja relativa de exportación de fresa, 1994-2016. Estados Unidos de América. Elaborado con datos de FAOSTAT (2019); FAS-USDA (2019). 


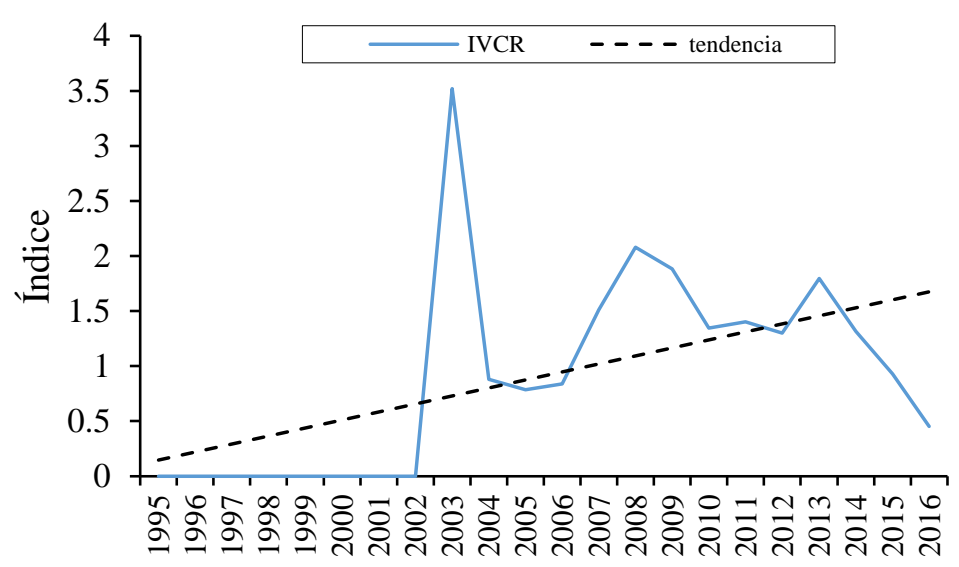

Figura 10. Índice de ventaja comparativa revelada de fresa, 1994-2016. Estados Unidos de América. Elaborado con datos de FAOSTAT (2019) y FAS-USDA (2019).

En México, durante el periodo de 1994 a 2016 el índice de ventaja relativa de exportación (IVRE) de fresa mostró una tendencia creciente, pasando de 0.4099 en 1994 a 1.005 en 2016, lo que indica que la fresa mexicana ha venido incrementando la competitividad en el mercado externo, además los valores positivos del IVRE sugieren la existencia de ventaja comparativa revelada y la especialización en las exportaciones de la fresa (Figura 11).

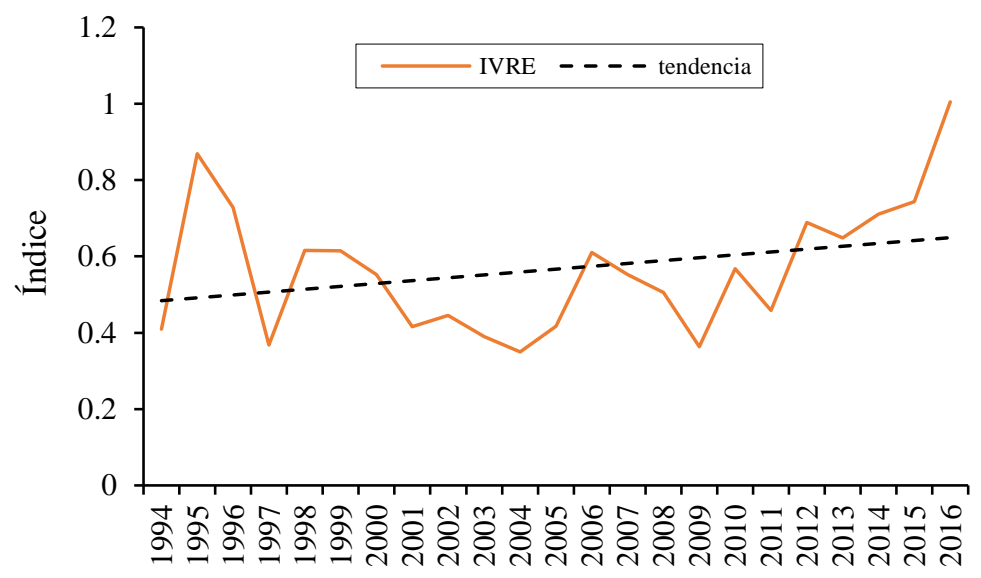

Figura 11. Índice de ventaja relativa de exportación de fresa, 1994-2016. México. Elaborado con datos de FAOSTAT (2019); FAS-USDA (2019).

Con relación al IVCR, en 1994 fue de 1.3987 y en 2016 de 2.9252, los valores más altos se ubican en los años 1995 y 2010 con 3.41 y 3.04 respectivamente; seguidos de 1996 con 2.96 y 2016 con 2.92, manteniendo, a lo largo del periodo el IVCR valores positivos (Figura 12). 


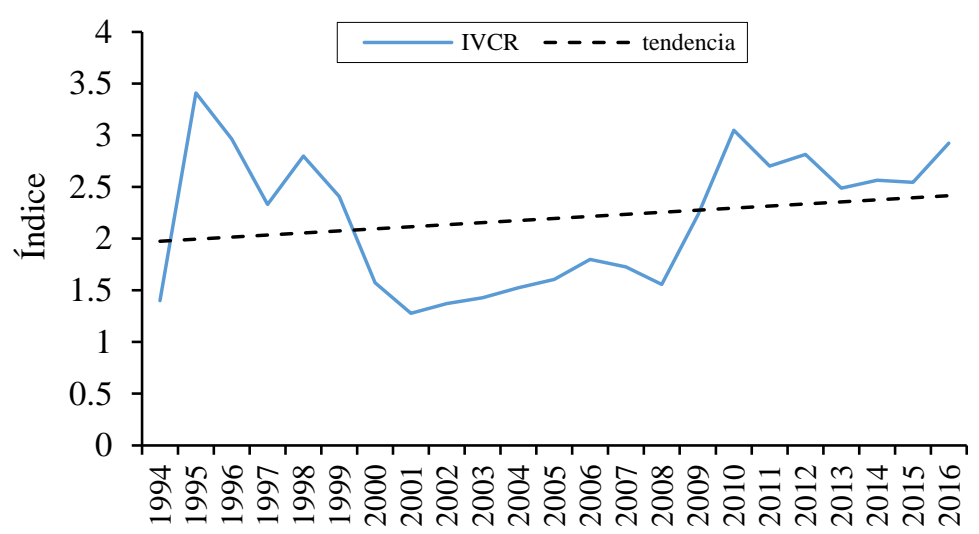

Figura 12. Índice de ventaja comparativa revelada de fresa, 1994-2016. México. Elaborado con datos de FAOSTAT (2019); FAS-USDA (2019).

Por lo que la fresa mexicana es un producto con ventaja comparativa revelada, con exportaciones superiores a las importaciones. Durante el periodo de 1994 a 2016, los valores del IVCR fueron mayores que uno, con tendencia creciente, reflejando desempeño positivo y competitivo de México en el mercado externo, con oportunidad de crecimiento, aprovechando la ventaja comparativa que tiene la fresa mexicana en el mercado internacional.

Los índices de ventaja relativa de exportaciones y de ventaja comparativa revelada señalan que existe especialización exportadora y ventaja comparativa en la exportación de fresa en el siguiente orden: España, México y Estados Unidos de América, reforzando y actualizando otros estudios realizados sobre el producto (Ayvar y González, 2011).

\section{Conclusiones}

Los resultados obtenidos con la aplicación de los índices de competitividad y de ventaja comparativa revelada, permitieron cumplir con el objetivo de identificar la competitividad de las exportaciones de fresa en el mercado exportador y de aceptar la hipótesis de que los índices de competitividad y ventaja comparativa revelada de las exportaciones de fresa mexicana son positivos y crecientes. Los mayores exportadores de fresa en el mundo son España, Estados Unidos de América y México. Las cantidades y tasas de crecimiento de las exportaciones de fresa de España, Estados Unidos de América y México de 1994 a 2016 fueron crecientes, reflejando expansión y competitividad del producto.

Por otro lado, los índices o coeficiente de exportación y de ventaja relativa de exportación positivos, mayores que cero, refleja por un lado, que los tres países tienen excedentes de producción que se destinan a la exportación y por otro lado, los valores de los índices de exportación y de ventaja relativa de exportación reflejan que España y México, exportan una mayor proporción y EE.UU. una menor proporción de la producción, resaltando una mayor especialización exportadora en fresa de España y México y una menor especialización exportadora de fresa de Estados Unidos de América, que destina más del noventa por ciento de la producción para el mercado interno. 
Asimismo, los índices de ventaja comparativa revelada positivos, mayores que cero, reflejan que los tres países son competitivos y tienen ventaja comparativa revelada, siendo España el más competitivo, seguido de México y finalmente, de Estados Unidos de América.

Las tasas de crecimiento positivas de la producción y exportación y los índices de competitividad y de ventaja comparativa revelada positivos de las exportaciones, reflejan que la producción y exportación de fresa se encuentra en expansión, es competitiva y tiene ventaja comparativa revelada en España, México y Estados Unidos de América, por lo que es recomendable la ampliación del cultivo, el incremento de la producción y el aumento de la exportación.

\section{Literatura citada}

Arias, J. y Segura, O. 2004. Índice de ventaja comparativa revelada: un indicador del desempeño y de la competitividad productivo-comercial de un país. Instituto Interamericano de Cooperación para la Agricultura. Costa Rica. 10 p.

Avendaño, B. D. 2008. Globalización y competitividad en el sector hortofrutícola: México, el gran perdedor. México. El Cotidiano. 147:91-98.

Ávila, A. y González, D. de J. 2012. La competitividad de las fresas (Fragaria spp.) mexicanas en el mercado nacional, regional y de Estados Unidos. México. Agricultura, Sociedad y Desarrollo. 9(1):17-27.

Ayvar, F. J. y González, J. 2011. Competitividad revelada del sector agrícola mexicano: El caso de la fresa. In: La competitividad como factor de éxito. Sánchez, J.; Gaytán, J. y Vázquez, M. (coords), $1^{\text {ra }}$ ed. Red Internacional de Investigadores en Competitividad. Guadalajara, México. 167-186 pp.

Ayvar, F. J.; Navarro, J. C. L. y Delfín, O. V. 2018. Competitividad y productividad del sector agropecuario mexicano en APEC, 1980-2015. México. Portes. Revista Mexicana de Estudios sobre la Cuenca del Pacífico. 12(23):7-30.

Balassa, B. (1965). "Trade liberalization and revealed comparative advantage". The Manchester School of Economics and Social Studies. 33(2):92-123. https://doi.org/10.1111/j.14679957.1965.tb00050.x.

Chordi, S. 2013. Contenido fenólico y capacidad antioxidante de fresa mínimamente procesada sometida a tratamientos de conservación por pulsos de luz de alta intensidad. Tesis. Facultad de Medicina, Universidad de Lleida, España. 45 p.

Contreras, J. M. 1999. La competitividad de las exportaciones mexicanas de aguacate: un análisis cuantitativo. México. Revista Chapingo Serie Horticultura. 5(Especial):393-400.

Daza, L. G. 2014. Determinación de la competitividad en países de América Latina: aplicación de un nuevo método. México. Perfiles Latinomericanos. 22(44):219-234.

De Pablo, J. y Giacinti, M. A. 2011. Competitividad en el comercio internacional vs ventajas comparativas reveladas (VCR): ensayo sobre exportaciones de manzanas de América del Sur. México. Revista Mexicana de Economía Agrícola y de los Recursos Naturales (REMECAREN). 6(1):49-82.

FAOSTAT. 2019. The statistics division of the Food and Agriculture Organization of the United Nations: http://faostat3.fao.org/faostat-gateway/go/to/download/T/TP/S.

FAS-USDA. 2019. Foreign Agricultural Service-United States Department of Agriculture. http://www.fas.usda.gov/. 
González, R. 2017. Competitividad de las exportaciones: un análisis teórico de indicadores de ventajas comparativas reveladas y su aplicación a los datos de comercio de Paraguay. Documento de trabajo N. 1. Subsecretaria de Estado de Economía, Ministerio de Hacienda. Paraguay. $35 \mathrm{p}$.

Heredia, J. y Huarachi, J. 2009. El índice de la ventaja comparativa revelada (VCR) entre el Perú y los principales exportadores del mundo. El caso de la Región Lambayeque. Perú. Journal of Economics, Finance and Administrative Science. 14(26):27-55.

Mortimore, M.; Bonifaz, J. L. y Duarte de Oliveira, J. L. 1997. La competitividad internacional: un análisis de las experiencias de Asia en desarrollo y América Latina. Naciones Unidas División de Desarrollo Productivo y Empresarial. Santiago de Chile. 69 p.

OCDE (Organisation for Economic Co-operation and Development). 1992. Technology and the Economy. The Key Relationships. OCDE. The Technology/Economy Programme 42. $328 \mathrm{p}$.

Restrepo, A.M.; Cortés, M. y Rojano, B. 2009. Determinación de la vida útil de fresa (Fragaria ananassa duch.) fortificada con vitamina E. Colombia. DYNA. 76(159):163-175.

Salazar, J. J. 2015. Estructura y evolución reciente de las ventajas comparativas de México y de sus estados. México. Revista de Ciencias Sociales de la Universidad Autónoma de Nuevo León. TRAYECTORIAS. 17(40):67-88.

Vargas, H. 2014. Desempeño competitivo de productos agropecuarios de Guatemala: una evaluación con base en las ventajas comparativas reveladas por el comercio internacional de 2000 a 2010. San José, Costa Rica. Instituto Interamericano de Cooperación para la Agricultura (IICA). 39 p.

Vollrath, T. 1991. A theoretical evaluation of alternative trade intensity measures of revealed comparative advantage. Germany. Review of World Economics. Weltwirtschaftliches Archiv. Institut für Weltwirtschaft (Kiel Institute for the World Economy). 127(2):265-280. 